\title{
Relação entre resultados da posturografia dinâmica "foam-laser", preocupação em cair e quedas em idosos praticantes de exercícios físicos
}

\author{
Relationship between results of the "foam laser" dynamic proturography, concern about falling \\ and falls in elderly practitioners physical exercise \\ Relación entre resultados de la posturografía dinámica "foam laser", preocupación por caídas y \\ caídas en el ancianos practicantes de ejercícios físicos
}

Recebido: 03/06/2021 | Revisado: 14/06/2021 | Aceito: 15/06/2021 | Publicado: 30/06/2021

Renata Silva Soares
ORCID: https://orcid.org/0000-0002-1921-4629
Universidade Federal do Rio Grande do Sul, Brasil
E-mail: renaatasoares@ gmail.com
Cristina Loureiro Chaves Soldera
ORCID: https://orcid.org/0000-0001-6417-2547
E-mail: cristina.soldera@ gmail.com
Maira Rozenfeld Olchik
Universidade Federal de Ciências da Saúde
ORCID: https://orcid.org/0000-0002-8732-9225
Universidade Federal do Rio Grande do Sul, Brasil
E-mail: mairarozenfeld@ @otmail.com
Andréa Kruger Gonçalves
ORCID: https://orcid.org/0000-0003-2772-3260
E-mail: andreakg@ drrgs.br
Universidade Federal do Rio Grande do Sul, Brasil
Alexandre Hundertmarck Lessa
ORCID: https://orcid.org/0000-0002-3083-8934
Universidade Federal do Rio Grande do Sul, Brasil
E-mail: alexandrehl@ gmail.com
Adriane Ribeiro Teixeira
ORCID: https://orcid.org/0000-0003-4242-1666
Universidade Federal do Rio Grande do Sul, Brasil
E-mail: Adriane.teixeira@ gmail.com

\begin{abstract}
Resumo
Objetivo: Verificar a relação entre os resultados da Posturografia Dinâmica "Foam-Laser", quedas e preocupação em cair em idosos praticantes de exercício físico. Metodologia: Estudo transversal, observacional, descritivo. A população foi composta por idosos de ambos os sexos, praticantes regulares de exercícios físicos. Os idosos responderam a anamnese, o instrumento Falls Efficacy Scale International (FES-I-Brasil), para verificar a preocupação em cair e foram avaliados por meio da Posturografia Dinâmica "Foam-Laser" para avaliar os sistemas de manutenção do equilíbrio corporal. Resultados: Foram incluídos na amostra 109 indivíduos, dos quais 89 são do sexo feminino e 20 do masculino, com média de idade de 72,1 $\pm 6,2$ anos. Os resultados do FES-I-Brasil evidenciaram mínima preocupação em cair, independente da idade. Entre os idosos caidores $(24,8 \%)$ a média da pontuação no FESI-Brasil foi de $24,7 \pm 9,40$ pontos e entre os não caidores $(75,2 \%)$ foi de $23,9 \pm 6,42$ pontos. No que se refere à posturografia, constatou-se que os idosos de 80 anos ou mais tiveram um pior desempenho na prova TOS V (Teste de Organização Sensorial) e predominância vestibular quando comparados aos idosos de 60 a 69 anos. Verificou-se que os idosos avaliados demonstraram valores médios inferiores ao ponto de corte proposto na literatura especializada em todos os testes de análise sensorial quando comparados aos valores de normalidade encontrados na literatura, exceto na prova TOS V por idosos de faixa etária menor. Conclusão: Não se observou relação entre as avaliações realizadas, independentemente da ocorrência ou não de quedas entre os idosos avaliados.
\end{abstract}

Palavras-chave: Envelhecimento; Equilíbrio; Quedas.

\section{Abstract}

Purpose: To verify the relationship between the results of the "Foam-Laser" Dynamic Posturography, falls and concern about falling in elderly practitioners of physical exercise. Methodology: Cross-sectional, observational, descriptive study. The population consisted of elderly people of both sexes, regular practitioners of physical exercise. The elderly answered the anamnesis, the instrument Falls Efficacy Scale International (FES-I-Brazil), to verify the 
concern with falling and were evaluated using the "Foam-Laser" Dynamic Posturography to assess the systems for maintaining body balance. Results: 109 individuals were included in the sample, of which 89 are female and 20 are male, with a mean age of $72.1 \pm 6.2$ years. The results of the FES-I-Brasil showed minimal concern about falling, regardless of age. Among elderly fallers $(24.8 \%)$, the mean score on the FES-I-Brazil was $24.7 \pm 9.40$ points and among non-fallers $(75.2 \%)$ it was $23.9 \pm 6.42$ points. With regard to posturography, it was found that the elderly aged 80 years or more had a worse performance in the TOS V test (Sensory Organization Test) and vestibular predominance when compared to the elderly aged 60 to 69 years. It was found that the evaluated elderly showed mean values lower than the cutoff point proposed in the specialized literature in all sensory analysis tests when compared to the normal values found in the literature, except in the TOS V test for younger elderly people. Conclusion: There was no relationship between the assessments performed, regardless of the occurrence or not of falls among the elderly assessed.

Keywords: Aging; Balance; Falls.

\section{Resumen}

Objetivo: Verificar la relación entre los resultados de la Posturografía Dinámica "Foam-Láser", las caídas y la preocupación por las caídas en ancianos practicantes de ejercicio físico. Metodología: Estudio descriptivo, observacional y transversal. La población estuvo constituida por personas mayores de ambos sexos, practicantes habituales de ejercicio físico. Los ancianos respondieron la anamnesis, el instrumento Falls Efficacy Scale International (FES-I-Brasil), para verificar la preocupación por las caídas y fueron evaluados mediante la Posturografía Dinámica "Foam-Laser" para evaluar los sistemas de mantenimiento del equilibrio corporal. Resultados: se incluyeron en la muestra 109 individuos, de los cuales 89 son mujeres y 20 son hombres, con una edad promedio de 72,1 \pm 6,2 años. Los resultados de la FES-I-Brasil mostraron una mínima preocupación por las caídas, independientemente de la edad. Entre los ancianos con caída (24,8\%), la puntuación media en la FES-I-Brasil fue de $24,7 \pm 9,40$ puntos y entre los no caídos $(75,2 \%)$ fue de $23,9 \pm 6,42$ puntos. Con respecto a la posturografía, se encontró que los ancianos de 80 años o más tenían peor desempeño en la prueba TOS V (Test de Organización Sensorial) y predominio vestibular en comparación con los ancianos de 60 a 69 años. Se encontró que los ancianos evaluados mostraron valores medios inferiores al punto de corte propuesto en la literatura especializada en todas las pruebas de análisis sensorial cuando se compararon con los valores normales encontrados en la literatura, excepto en la prueba TOS V para ancianos más jóvenes. Conclusión: No hubo relación entre las evaluaciones realizadas, independientemente de la ocurrencia o no de caídas entre los ancianos evaluados.

Palabras clave: Envejecimento; Equilibrio; Caidas.

\section{Introdução}

Nas últimas décadas a expectativa de vida aumentou significativamente, alcançando a média de, aproximadamente, 70 anos em 2014 nos países em desenvolvimento e de 80 anos nos países desenvolvidos (Jin et al., 2014). Segundo as Nações Unidas (2019), estima-se que até 2050 a população idosa irá duplicar, e poderá chegar a 1,5 bilhões de pessoas. No Brasil, em 2017, a população idosa representava 10\% da população total (Cardoso, Dietrich \& Souza, 2021). Nesse caso, é necessário que seja cada vez maior a preocupação da comunidade científica em realizar estudos que contemplem abordagens com os indivíduos nesta faixa etária, considerando que o aumento na sobrevida implica em mudanças permanentes em políticas sociais, por exemplo (Cardoso, Dietrich \& Souza, 2021).

Com o envelhecimento o corpo tem maior suscetibilidade a alterações na integração entre os sistemas sensorial e motor. Este fato interfere diretamente na manutenção de uma postura estável (equilíbrio estático), ou em movimento (equilíbrio dinâmico), de maneira harmônica e precisa (Hueb \& Feliciano, 2012; Mujedci, Aksoy \& Atas, 2012; Hauser, Cardoso \& Mazo, 2020). O comprometimento do sistema postural do indivíduo pode ocorrer devido a alteração proprioceptiva (percepção da postura e da movimentação do corpo), vestibular (posição e movimento da cabeça, incluindo aceleração linear e angular), ou visual (relações espaciais e percepção do ambiente), o que causa desordens de equilíbrio e quedas, originando preocupação em cair, o que interfere negativamente na qualidade de vida. Estudos demonstram que a prática de exercícios físicos auxilia para que se tenha um envelhecimento saudável, amenizando essas possíveis alterações e auxiliando na manutenção da capacidade funcional (Kirk-Sanchez \& McGough, 2014; Scianni et al, 2019). A prática de exercícios físicos pode neutralizar os efeitos do envelhecimento na aferência vestibular, particularmente por aumentar ou manter a sensibilidade desta informação sensorial. Além disso, essa estimulação pode gerar mecanismos de compensação melhores e mais rápidos, 
gerando maior adequação dos reflexos vestíbulo-oculares e viso-oculares. Todos esses efeitos positivos sobre os sistemas periféricos e central levam a ajustes corporais mais precisos por estar o reflexo vestibuloespinal mais bem adaptado à desestabilização (Gauchard et al., 2003).

A prevalência de quedas no Brasil é de 30\%, tanto em idosos na comunidade, como institucionalizados (Sandoval et al., 2013). Entre os idosos que vivenciaram uma queda, avalia-se que cerca de $50 \%$ terão novos episódios, $10 \%$ terão fraturas e mais de 20\% irão reduzir suas ocupações por temer cair novamente (Siqueira et al., 2011; Amorim et al., 2021). Além disso, estudo nacional demonstrou que as mulheres apresentam maior chance de cair (Amorin et al., 2021).

Considerando a prevalência de distúrbios do equilíbrio e de quedas em idosos, é importante que tais aspectos sejam avaliados. Existem diversas formas para avaliar o equilíbrio na população idosa, dentre elas, está a Posturografia Dinâmica "Foam-Laser", desenvolvida por Castagno (1994). Esta é uma técnica de custo acessível e fidedigna para a realização do Teste de Organização Sensorial (TOS). Trata-se de um aparato significativo para a quantificação da participação dos sistemas sensoriais acometidos nos distúrbios do equilíbrio corporal e permite caracterizar os impactos da idade sobre a função vestibular, e singularmente útil para avaliar a progressão da enfermidade e a efetividade dos métodos de reabilitação labirínticas utilizados.

Ainda com relação a quedas, diferentes estudos demonstram a pertinência clínica do medo de cair e a associação com a qualidade de vida desta população. Dessa forma, para verificação da preocupação em cair, foi desenvolvida por Tinetti, Richman \& Powell (1990) a escala para avaliação da autoeficácia relacionada às quedas denominada Falls Efficacy Scale (FES), sendo posteriormente modificado pela PRoFaNE (Prevention of Falls Network Europe), que incluiu seis itens ao questionário original, sendo a partir de então chamado de Falls Efficacy Scale-International (FES-I) (Yardley et al., 2005). A escala foi posteriormente adaptada e validada para o Brasil por Camargos et al. (2010) sendo denominada Falls Efficacy Scale International (FES-I_Brasil).

Considerando-se a prevalência de quedas em idosos e a relação destas com o medo de novas quedas, bem como a avaliação dos sistemas responsáveis pelo equilíbrio, elaborou-se este trabalho de pesquisa. Observou-se carência de estudos que relacionaram estes fatores e cujos resultados poderão colaborar para que os profissionais que atuam com indivíduos nesta faixa de idade possam usar como base para atividades de prevenção e tratamento. Partiu-se da pergunta seguinte pergunta de pesquisa: existe relação entre a ocorrência de quedas, preocupação em cair e resultados do TOS entre idosos caidores e não caidores? A hipótese dos pesquisadores é que existiria tal relação.

Assim, o presente trabalho tem como objetivo verificar a existência de relação entre os resultados do TOS, ocorrência de quedas e preocupação em cair em idosos praticantes de exercício físico.

\section{Metodologia}

Este estudo tem caráter do tipo transversal, observacional, descritivo. Foi aprovado pela Comissão de Pesquisa e pelo Comitê de Ética em Pesquisa da instituição de origem. Os idosos foram incluídos na amostra e avaliados somente após assinarem o Termo de Consentimento Livre e Esclarecido.

Foram critérios de inclusão na amostra: indivíduos de ambos os sexos, com idade igual ou maior a 60 anos, praticantes de diferentes tipos de exercícios físicos, de forma regular, em um programa de extensão na universidade. Foram excluídos: indivíduos com histórico de alteração de equilíbrio e marcha por alterações neurológicas ou motoras ou realização incompleta das avaliações. A forma de seleção da amostra está descrita a seguir.

Em um primeiro momento foi realizado o contato com todos os idosos que frequentavam um projeto de extensão na universidade para convite à participação na pesquisa. Na sequência foi realizada a anamnese em que foram verificados dados do perfil sócio-demográfico e de saúde dos idosos, incluindo questionamento sobre a ocorrência de quedas no último ano. 
Logo depois foi realizado o questionário sobre a preocupação em cair utilizando o instrumento FES-I-Brasil. Por meio de 16 questões que abordam atividades diárias distintas, tal preocupação é avaliada. Para cada questão o escore pode variar de um ponto (nenhuma preocupação em cair) a quatro pontos (extrema preocupação em cair). Assim, o escore total varia de 16 (ausência de preocupação) a 64 pontos (preocupação extrema) (Camargos et al., 2010). Os itens avaliados abrangem tarefas relacionadas ao controle postural, exigindo maior grau de dificuldade, e outras básicas, instrumentais e de socialização, que envolvem menor demanda física. Delbaere et al. (2010) sugerem que pontuações iguais ou inferiores a 22 pontos indicam baixa preocupação com quedas. Já pontuações entre 23 e 64 pontos podem evidenciar alta preocupação com quedas.

Após a realização dessas etapas realizou-se o Teste de Organização Sensorial com a Posturografia Dinâmica "foamlaser" (Castagno, 1994). Para a realização dessa avaliação utilizou-se uma cabine de dois metros de altura, feita com suporte de ferro. Cobrindo esse suporte, colocou-se um tecido de algodão com listras, claras e escuras. Colocou-se um suporte de ferro ao lado do idoso, com altura superior à cabine, e na parte superior deste, um painel com papel centimetrado. Na cintura do idoso foi colocado um cinto, onde foi presa uma caneta laser, que permaneceu na parte de trás do corpo, voltada para suporte de ferro em direção ao papel centimetrado. A observação do feixe de laser projetado no papel centimetrado permite a quantificação do deslocamento corporal no sentido ântero-posterior. Além disso, para a realização de parte do teste, também utilizou-se uma almofada de espuma, de cinquenta centímetros por cinquenta centímetros, com dez centímetros de espessura e densidade média (Ruwer, 2006; Soldera, 2013).

Os idosos foram posicionados dentro da cabine e orientados a permanecer em pé, com os pés unidos, em seis condições de conflito sensorial diferentes, para que se pudesse realizar o Teste de Organização Sensorial (TOS) (Rubin, 2002; Teixeira et al., 2015).

TOS I - o indivíduo mantém seus olhos abertos e fixos à frente mantendo-se sob uma superfície fixa e estável por aproximadamente 20 segundos;

TOS II - o indivíduo permanece com seus olhos fechados mantendo-se sob uma superfície fixa e estável por aproximadamente 20 segundos;

TOS III - o indivíduo permanece em uma superfície fixa e estável e a cabine é movida lentamente para traz em 10 segundos e retorna em 10 segundos para frente;

TOS IV - o indivíduo mantém seus olhos abertos e fixos à frente mantendo-se sob uma espuma de $10 \mathrm{~cm}$ de espessura e média densidade por aproximadamente 20 segundos;

TOS V - o indivíduo permanece com seus olhos fechados mantendo-se sob uma espuma de $10 \mathrm{~cm}$ de espessura e média densidade por aproximadamente 20 segundos;

TOS VI - o indivíduo mantém-se sob uma espuma de $10 \mathrm{~cm}$ de espessura e média densidade e a cabine é movida lentamente para traz em 10 segundos e retorna em 10 segundos para frente.

Durante a avaliação, três examinadores permaneceram ao lado dos idosos, sendo que dois observaram e anotaram os desvios corporais ântero-posteriores nos seus pontos máximos e um deles manteve-se ao lado dos indivíduos para auxiliar em caso de ocorrência de quedas, desvios muito grandes que pudessem levar a quedas ou relato de tontura. O deslocamento do laser no papel centimetrado também foi filmado para posteriormente ser analisado em conjunto com as anotações feitas pelos examinadores. A estratégia da filmagem não consta no teste original. Foi sugerida por Soldera (2013) para que os resultados obtidos fossem mais fidedignos.

Após a verificação do deslocamento máximo, em centímetros, utilizou-se a fórmula matemática computadorizada definida por Castagno (1994) para o cálculo do ângulo das oscilações. Quando não houve oscilações o escore foi de 100\%. Já 
nos casos de quedas ou desvios de oscilações angulares maiores do que $12,5^{\circ}$ o escore foi de $0 \%$ (Ruwer, 2006). A quantificação da participação dos sistemas sensoriais para a manutenção do equilíbrio, são realizadas com as seguintes verificações (Castagno, 1994):

- somatossensorial: TOS II/ TOS I;

- visual: TOS IV/ TOS I;

- vestibular: TOS V/ TOS I;

- preferência visual: TOS III + TOS VI/ TOS II + TOS V.

A análise estatística dos dados obtidos na pesquisa foi realizada no software SPSS (Statistical Package for the Social Sciences) versão 21.0. As variáveis contínuas foram descritas por média e desvio-padrão e as variáveis categóricas por frequências absolutas e relativas. Para comparar médias foram utilizados os testes t-Student ou Análise de Variância (ANOVA) em conjunto com o teste de Tukey. O nível de significância estatístico considerado foi de 5\% (p $\leq 0,05)$.

\section{Resultados}

A amostra inicial foi composta por 111 indivíduos, sendo um excluído por apresentar alteração motora em membros inferiores e um por queda durante a avaliação, não realizando o protocolo por completo. Assim, foram incluídos na amostra 109 indivíduos. Verificou-se que a média de idade foi de 72,1 $\pm 6,2$ anos, com maior prevalência de mulheres. Com relação a quedas, verificou-se que $24,8 \%$ dos avaliados relataram quedas no ano anterior a pesquisa. A caracterização da amostra é apresentada na Tabela 1.

Tabela 1 - Caracterização da amostra.

\begin{tabular}{|c|c|c|c|c|c|}
\hline Variáveis & $\begin{array}{l}\text { Amostra total } \\
(n=109)\end{array}$ & $\begin{array}{c}60 \text { a } 69 \text { anos } \\
(n=40)\end{array}$ & $\begin{array}{c}70 \text { a } 79 \text { anos } \\
(n=54)\end{array}$ & $\begin{array}{c}80 \text { anos ou mais } \\
(\mathrm{n}=15)\end{array}$ & $\mathrm{p}$ \\
\hline Idade (anos) - média \pm DP & $72,1 \pm 6,2$ & $65,6 \pm 2,9$ & $74,0 \pm 2,3$ & $82,3 \pm 3,0$ & - \\
\hline Sexo-n $(\%)$ & & & & & 0,266 \\
\hline Feminino & $89(81,7)$ & $30(75,0)$ & $45(83,3)$ & $14(93,3)$ & \\
\hline Masculino & $20(18,3)$ & $10(25,0)$ & $9(16,7)$ & $1(6,7)$ & \\
\hline Escolaridade (anos) - média \pm DP & $12,1 \pm 4,5$ & $12,4 \pm 4,8$ & $12,4 \pm 4,5$ & $10,2 \pm 3,5$ & 0,232 \\
\hline Quedas $-\mathrm{n}(\%)$ & & & & & 0,269 \\
\hline Sim & $27(24,8)$ & $7(17,5)$ & $17(31,5)$ & $3(20,0)$ & \\
\hline Não & $82(75,2)$ & $33(82,5)$ & $37(68,5)$ & $12(80,0)$ & \\
\hline
\end{tabular}

Legenda: DP - desvio padrão; $\mathrm{n}$ - número; \% - porcentagem; $\mathrm{p}$ - valor de $\mathrm{p}$

Fonte: Autores.

Constatou-se correlação significativa no desempenho da análise sensorial (TOS) analisada por faixa etária. Os idosos de 80 anos ou mais tiveram um pior desempenho no TOS V e predominância vestibular quando comparados aos idosos de 60 a 69 anos (Tabela 2). Os valores obtidos também são inferiores aos padrões de normalidade, exceto no TOS V em que os idosos de 60 a 69 anos obtiveram melhor desempenho (Gráfico 1). 
Tabela 2 - Achados sobre TOS por faixa etária na amostra estudada.

\begin{tabular}{|c|c|c|c|c|c|}
\hline \multirow[t]{2}{*}{ Escala TOS } & $\begin{array}{c}\text { Amostra total } \\
\quad(n=109)\end{array}$ & $\begin{array}{c}60 \text { a } 69 \text { anos } \\
(n=40)\end{array}$ & 70 a 79 anos $(n=54)$ & 80 anos ou mais $(n=15)$ & \multirow[t]{2}{*}{$\mathrm{p}$} \\
\hline & média \pm DP & média \pm DP & média \pm DP & média \pm DP & \\
\hline I & $76,0 \pm 9,7$ & $75,8 \pm 8,2$ & $75,8 \pm 11,3$ & $77,3 \pm 6,8$ & 0,859 \\
\hline II & $67,7 \pm 12,4$ & $70,5 \pm 11,9$ & $66,1 \pm 12,7$ & $66,1 \pm 12,5$ & 0,200 \\
\hline III & $55,6 \pm 19,9$ & $55,4 \pm 18,2$ & $55,4 \pm 18,7$ & $57,0 \pm 28,7$ & 0,957 \\
\hline IV & $72,2 \pm 12,9$ & $73,1 \pm 12,8$ & $71,5 \pm 14,1$ & $72,0 \pm 8,2$ & 0,844 \\
\hline V & $60,9 \pm 16,7$ & $66,7 \pm 10,1^{b}$ & $58,1 \pm 18,3^{\mathrm{ab}}$ & $55,8 \pm 21,2^{\mathrm{a}}$ & $0,020^{*}$ \\
\hline VI & $44,3 \pm 23,6$ & $47,7 \pm 23,2$ & $44,0 \pm 23,2$ & $36,1 \pm 25,6$ & 0,265 \\
\hline Média TOS & $62,1 \pm 13,0$ & $64,2 \pm 11,7$ & $61,3 \pm 13,4$ & $59,3 \pm 15,0$ & 0,389 \\
\hline SOM & $90,1 \pm 17,9$ & $94,4 \pm 15,2$ & $88,2 \pm 19,8$ & $85,6 \pm 15,9$ & 0,141 \\
\hline VIS & $95,8 \pm 18,3$ & $96,9 \pm 17,5$ & $95,6 \pm 10,4$ & $93,6 \pm 11,5$ & 0,831 \\
\hline VEST & $80,7 \pm 24,2$ & $88,0 \pm 12,9^{\mathrm{b}}$ & $77,9 \pm 28,9^{\mathrm{ab}}$ & $71,3 \pm 24,8^{\mathrm{a}}$ & $0,034^{*}$ \\
\hline PREF & $76,8 \pm 30,5$ & $72,2 \pm 26,1$ & $80,9 \pm 31,3$ & $74,3 \pm 38,0$ & 0,368 \\
\hline
\end{tabular}

Legenda: $\mathrm{n}$ - número; DP - desvio padrão; TOS - Teste de Organização Sensorial.

${ }^{\mathrm{a}, \mathrm{b}}$ Letras iguais não diferem pelo teste de Tukey a 5\% de significância.

$*$ valores significativos.

Fonte: Autores.

Figura 1 - Valores médios nas análises sensoriais do TOS nos grupos etários e valores de normalidade.

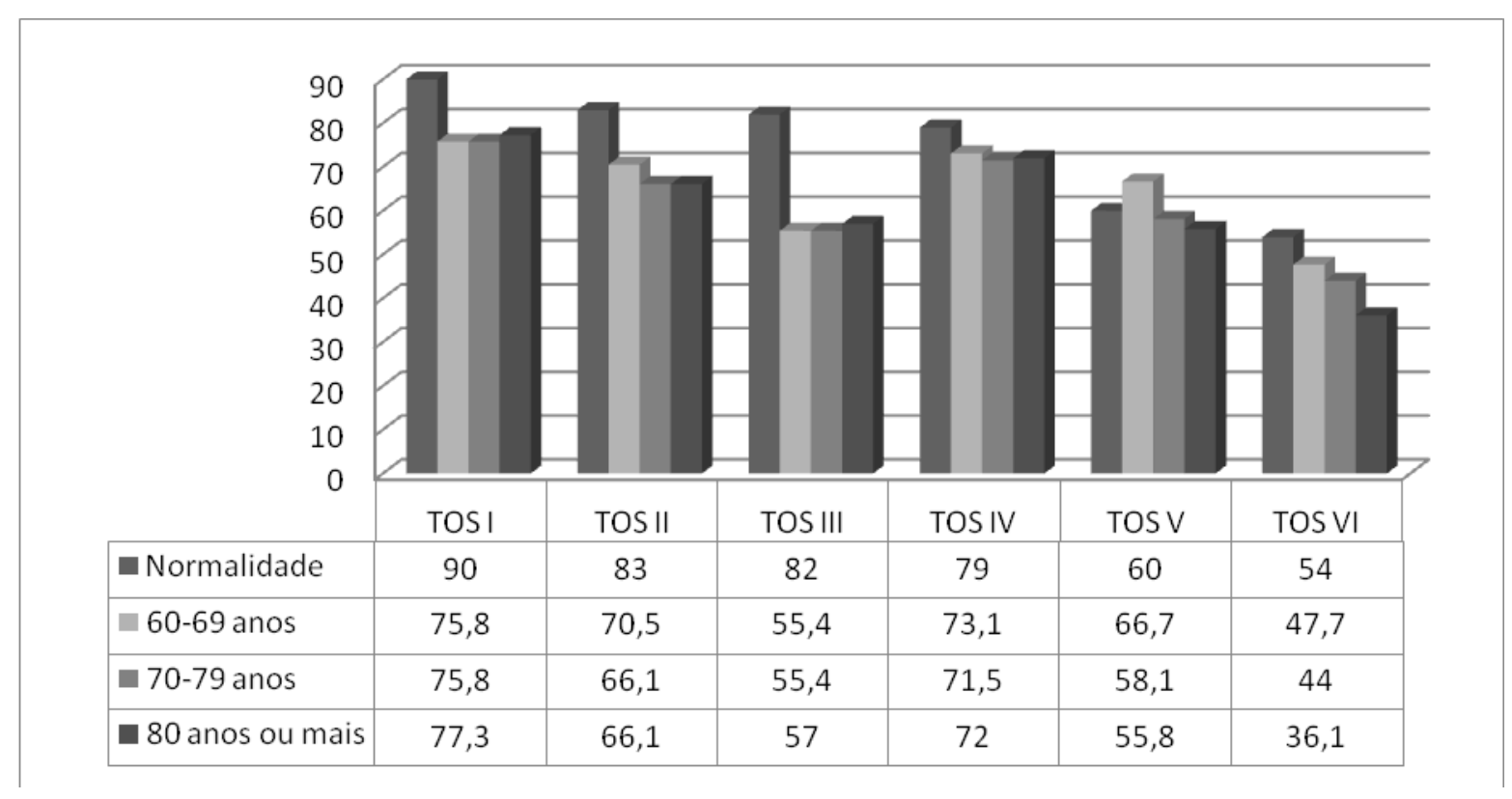

Legenda: TOS - Teste de Organização Sensorial.

Fonte: Autores. 
Os resultados obtidos na escala FES-I-Brasil evidenciaram que a maior parte dos participantes do estudo apresentava pouca preocupação em cair (16 a 22 pontos), independentemente da faixa etária (Tabela 3 ).

Tabela 3 - Escore na escala FES-I-Brasil por faixa etária na amostra estudada.

\begin{tabular}{lcccc}
\hline Pontuação FES - n (\%) & Amostra total & 60 a 69 anos & 70 a 79 anos & 80 anos ou mais \\
\hline $16-22$ & $58(53,2)$ & $22(55,0)$ & $30(55,6)$ & $6(40,0)$ \\
$23-30$ & $34(31,2)$ & $13(32,5)$ & $15(27,8)$ & $6(40,0)$ \\
31 ou mais & $17(15,6)$ & $5(12,5)$ & $9(16,7)$ & $3(20,0)$ \\
\hline
\end{tabular}

Legenda - FES - Escala Falls Eficacy Scal; n- número; \% - porcentagem.

Fonte: Autores.

Quando analisados os dados TOS e do FES-I-Brasil em comparação com o histórico que quedas no último ano, não houve correlação significativa (Tabela 4).

Tabela 4 - Resultados do TOS e FES-I-Brasil na amostra estudada com e sem quedas no último ano.

\begin{tabular}{|c|c|c|c|}
\hline & $\begin{array}{c}\text { Presença de quedas } \\
(\mathrm{n}=27)\end{array}$ & $\begin{array}{l}\text { Ausência de quedas } \\
\qquad(\mathrm{n}=82)\end{array}$ & $\mathrm{p}$ \\
\hline & média \pm DP & média \pm DP & \\
\hline \multicolumn{4}{|c|}{ Teste de Organização Sensorial } \\
\hline TOS - I & $75,5 \pm 12,1$ & $76,2 \pm 8,8$ & 0,73 \\
\hline TOS - II & $69,4 \pm 14,9$ & $67,2 \pm 11,6$ & 0,42 \\
\hline TOS - III & $55,4 \pm 19,5$ & $55,7 \pm 20,2$ & 0,94 \\
\hline TOS - IV & $73,3 \pm 9,4$ & $71,8 \pm 13,9$ & 0,61 \\
\hline TOS $-\mathrm{V}$ & $64,4 \pm 19,4$ & $59,8 \pm 15,6$ & 0,21 \\
\hline TOS - VI & $46,3 \pm 25,3$ & $43,6 \pm 23,2$ & 0,61 \\
\hline Média TOS & $63,9 \pm 13,2$ & $61,5 \pm 13,0$ & 0,40 \\
\hline SOM & $93,1 \pm 22,0$ & $89,2 \pm 16,3$ & 0,32 \\
\hline VIS & $98,5 \pm 15,2$ & $94,9 \pm 19,2$ & 0,37 \\
\hline VEST & $86,2 \pm 34,3$ & $78,9 \pm 19,8$ & 0,17 \\
\hline PREF & $77,1 \pm 34,2$ & $76,7 \pm 29,4$ & 0,95 \\
\hline FES-I-Brasil & $24,78 \pm 9,40$ & $23,96 \pm 6,42$ & 0,56 \\
\hline
\end{tabular}

Legenda: $\mathrm{n}$ - numero; DP - desvio padrão; TOS - Teste de Organização Sensorial; $\mathrm{p}$ - valor de p; SOM somatossensorial ; VIS - visual; VEST - vestibular; PREF - preferência visual; FES-I-Brasil - Escala Falls Efficacy Scale - versão em português brasileiro Fonte: Autores. 
A análise da entre os resultados do TOS com a preocupação em cair não evidenciou associação significativa (Tabela $5)$.

Tabela 5 - Associação entre resultados ao TOS e escores do FES-I-Brasil.

\begin{tabular}{lll}
\hline TOS & \multicolumn{2}{l}{ FES-I-Brasil } \\
\cline { 2 - 3 } & Coeficiente de correlação de Pearson (r) & $\mathrm{p}$ \\
\hline I & 0,028 & 0,776 \\
II & 0,058 & 0,546 \\
III & $-0,146$ & 0,130 \\
IV & 0,010 & 0,914 \\
V & 0,049 & 0,610 \\
VI & $-0,131$ & 0,175 \\
Média TOS & $-0,094$ & 0,330 \\
SOM & $-0,004$ & 0,965 \\
VIS & $-0,020$ & 0,836 \\
VEST & 0,030 & 0,755 \\
PREF & $-0,184$ & 0,056 \\
\hline
\end{tabular}

Legenda: TOS - Teste de Organização Sensorial; SOM - somatossensorial; VIS - visual; VEST vestibular; PREF - preferência visual; $\mathrm{p}$ - valor de $\mathrm{p}$ Fonte: Autores.

\section{Discussão}

Os dados do presente estudo permitem observar que a amostra, formada predominantemente por mulheres, é comparável a estudos anteriores (Berlezi et al., 2016, Soldera, 2013; Teixeira-Leite \& Manhães, 2012), podendo ser explicado pela maior longevidade dessas em relação aos homens. Além disso, o estudo foi feito com idosos participantes de programa de extensão universitária, e pesquisas anteriores com este público evidenciam um número maior de mulheres (Galisteu et al., 2006; Fiorio \& Meneghini, 2018; Teixeira, Cardoso \& Olchik, 2021). Deve-se considerar, também, que os dados do município no qual a pesquisa foi realizada (PROCEMPA, 2015), indicam que a população idosa do sexo feminino é maioria no município $(62,25 \%)$.

A média de escolaridade dos idosos da presente amostra se mostrou mais elevada quando comparada com o equivalente ao Rio Grande do Sul e Brasil. No Estado, a média segundo o último levantamento do IBGE (2015), é de 4,1 anos de escolaridade, três vezes menor do que os achados desta pesquisa. Cardoso, Dietrich \& Souza (2021) referem que em 2017 a média de escolaridade dos idosos com 75 anos ou mais era de 4,6 anos de estudo, aumentando para 6,3 anos quando eram analisados os dados dos indivíduos entre 65 e 74 anos. Uma das hipóteses relacionadas ao nível de escolaridade ser tão elevado na amostra, deve-se ao fato de o projeto de extensão no qual os idosos participam ter, entre seus integrantes, pessoas que fizeram parte do corpo administrativo ou docente da universidade, ou professoras aposentadas da rede pública. Além disso, o local de realização do projeto é situado em um bairro classe média-alta de Porto Alegre. Dados da PROCEMPA (2015) demonstram que na região onde o projeto é desenvolvido, a taxa de analfabetismo é de $0,78 \%$ e o rendimento médio dos responsáveis por domicílio é de 7,45 salários-mínimos. Não se observou diferenças significativas entre os idosos e longevos no quesito escolaridade.

Verificou-se que 24,8\% dos idosos apresentaram pelo menos um episódio de queda no último ano, estando a maior parte dos caidores na faixa etária de 70 a 79 anos. A prevalência de quedas é comparável a outros estudos, que consideram que a prevalência de quedas entre idosos está em aproximadamente 30\% dos casos (Cruz, Duque \& Leite, 2017; Vieira et al., 
2018). A queda é um fenômeno amplamente descrito como um dos principais causadores de internações, fraturas, incapacidades e morte na idade avançada (Myerrs, Young \& Langlois, 1996). Os dados obtidos corroboram achados da literatura nessa área de estudo (Lee et al., 2013). Lopes et al., (2009) (2012), ao analisar idosos, encontraram associação entre ocorrência de quedas e idade. Destaca-se, contudo, que estudos (Cadore et al., 2013; Gillespie et al., 2012) apontam que programas de exercícios físicos semanais melhoram a atividade neuromuscular, a massa muscular, a força e a capacidade funcional, diminuindo o risco de quedas nesta população. Este dado não foi observado na amostra avaliada, uma vez que o número de quedas foi semelhante aos estudos citados, contrariando o que era esperado pelos pesquisadores.

O presente estudo verificou que em todas as condições do Testes de Organização Sensorial (TOS) os idosos da amostra ficaram abaixo dos valores de referência descritos por Castagno (1994). Este dado corrobora achados de outro estudo com este mesmo teste em idosos (Ruwer, 2002), e pode ocorrer porque adultos e idosos utilizam-se de estratégias distintas para a manutenção do equilíbrio (Borel \& Alescio-Lautier, 2014). Destaca-se, contudo, que quando os valores de TOS são comparados com estudos que analisam os dados dos idosos separadamente, verifica-se que os valores obtidos em cada um dos TOS são superiores (Teixeira et al., 2015). No que se refere ás análises de cada um dos sistemas e o estudo citado anteriormente, nossos valores são próximos aos obtidos no sistema visual. Nos demais testes nossos resultados são inferiores.

À vista disso, confirma-se a diferença entre as faixas etárias (Teixeira et al., 2015) e a necessidade de se estabelecer valores de referência das análises sensoriais do TOS, de acordo com as faixas etárias específicas, para a avaliação ser descrita com melhor propriedade (Ruschel et al., 2019).

O estudo de Soldera (2013) encontrou diferenças entre idosos nas análises do TOS. O desempenho entre os longevos foi considerado pior quando comparado com o desempenho dos idosos jovens. Este padrão foi encontrado neste estudo apenas no TOS V no qual há alteração do sistema somatossensorial e ausência da visão. Estes achados evidenciam alteração do equilíbrio corporal com o envelhecimento (Cadore et al., 2013). Há indícios de que para se manter a referência postural é necessária $70 \%$ da informação somatossensorial, $10 \%$ visual e $20 \%$ vestibular. No momento que se permanece em uma superfície oscilante, a carga da informação sensorial vestibular e visual aumenta, e reduz a interação sobre a superfície da entrada somatossensorial. Consequentemente, pode-se prever que maiores oscilações sejam nos TOS V e VI, tendo em vista que mais de um sistema sensorial é impactado em uma mesma avaliação (Peterka, 2002).

Além disso, os resultados obtidos na presente pesquisa evidenciam que, exceto na posição V (pés sobre a almofada e olhos fechados) e na análise vestibular não houve associação entre os resultados do TOS e a faixa etária. Este dado pode ser explicado pelo fato de que os idosos realizavam atividade física orientada regularmente (Gillespie et al., 2012, Cadore et al., 2013). Os exercícios físicos podem auxiliar na diminuição da fragilidade óssea, das dores articulares e de alteração das funções relacionadas ao equilíbrio corporal que acontecem em função do envelhecimento (Pedrinelli, Garcez-Leme \& Nobre, 2009). Pesquisadores referem que a realização de atividade física regular pode prevenir a ocorrência de quedas em idosos, enfatizando que o sedentarismo leva a menor mobilidade e maior tendência a quedas (Guimarães et al., 2004).

A escala FES-I-Brasil (Camargos et al., 2010) avalia a preocupação em cair. Quanto maior a pontuação na escala, maior a preocupação em cair. Considerando a amostra total, verificou-se que a maioria dos idosos $(53,2 \%)$ apresentou pontuações entre 16 e 22 pontos, demonstrando baixa preocupação com quedas. Já na análise por faixa etária, constatou-se a confirmação deste dado, exceto nos idosos com 80 anos ou mais, quando $40 \%$ dos avaliados apresentaram pontuações entre 16 e 22 pontos e uma mesma porcentagem entre 23 e 30 pontos (alta preocupação com quedas). A pontuação obtida foi semelhante à observada por outros pesquisadores (Cruz, Duque \& Leite, 2017), mas os resultados diferem destes autores que observaram correlação entre os resultados do FES-I-Brasil e a idade.

A média da pontuação do FES-I-Brasil e os resultados do TOS, considerando idosos caidores e não caidores, não apresentou associações significativas. Estes dados foram diferentes do esperado pelos pesquisadores, uma vez que a literatura 
aponta que a porcentagem de idosos que se preocupam em cair varia de 21 a $85 \%$ entre os que vivenciaram uma queda anteriormente, e de 33 a 46\% entre a população não caidora (Gillespie et al., 2012). Idosos com menor medo em cair demonstram ter maior força, equilíbrio e agilidade quando comparados àqueles com maior medo de cair. Dessa forma, ações voltadas para a otimização dessas habilidades podem ser uma possibilidade para a atenuação do medo de cair nessa população (Hauser et al., 2015). Estudos demonstram que quanto menos o indivíduo se preocupa em cair maior sua capacidade de autonomia/ independência, e em vista disso, executam suas atividades de vida diária com maior confiança (Hornyak et al., 2013). Essas evidências podem justificar o motivo pelo qual a maioria dos idosos praticantes de exercício físico do presente estudo apresentaram preocupação mínima em cair, independente do seu desempenho nas análises sensoriais ou condição de caidor.

A análise entre os resultados do TOS e do FES-I-Brasil evidenciaram ausência de correlação, corroborando os achados do estudo, de que a preocupação em cair não é um fato entre os idosos avaliados, mesmo com valores ao TOS abaixo do ponto de corte estabelecido pelo autor do instrumento. Talvez isto possa novamente ser explicado pela prática de exercícios físicos, considerando-se que o controle postural é porção complementar do sistema de controle motor humano, que origina estabilidade e conjuntura para o movimento. Além disso, o mesmo é uma complexa função sensório-motora que requer a junção das informações dos sistemas vestibular, proprioceptivo e visual para fornecer uma reação motora que possibilite o equilíbrio em atividades tanto estáticas, quanto dinâmicas (Teixeira, 2013). Assim, como descrito anteriormente, para minimizar possíveis declínios oriundos do envelhecimento, especialmente os relacionados para a manutenção do equilíbrio, a prática regular de atividades e exercícios físicos deve ser indicada (Cadore et al., 2013).

Como limitações do estudo, podemos destacar o tipo de estudo realizado, transversal. Seria importante a possibilidade de estudos longitudinais para a avaliação do equilíbrio em idosos. Uma outra limitação seria a impossibilidade de analisar ou realizar relação entre o tipo de exercício físico realizada pelos idosos. Devido ao fato dos mesmos praticarem diferentes tipos de exercícios e inclusive realizarem mudanças do tipo de exercícios ao longo do tempo, não sendo possível relacionar o tipo de exercício, o número de práticas por semana e os resultados obtidos.

\section{Conclusão}

De acordo com os resultados encontrados no presente estudo, não foram observadas relações entre os resultados das avaliações realizadas. A prática de exercícios físicos parece auxiliar os idosos na manutenção do equilíbrio corporal.

Observou-se que os idosos avaliados não apresentaram maior preocupação em cair, independente de sua condição de caidor. Além disso, o caráter de caidor não influenciou nos desempenhos das análises sensoriais. As médias obtidas no TOS estão abaixo dos padrões de normalidade descritas pelo autor da avaliação, destacando-se, assim, a necessidade de se estabelecer pontos de corte específicos por idade.

\section{Agradecimentos}

Os autores agradecem a todos os idosos que aceitaram participar do estudo.

\section{Referências}

Amorim, J. S. C et al. (2021). Prevalência de queda grave e fatores associados em idosos brasileiros: resultado da Pesquisa Nacional de Saúde, 2013. Ciência e Saúde Coletiva, 26(1), 185-196. https://doi.org/10.1590/1413-81232020261.30542018

Berlezi, E. M. et al. (2016). Analysis of the functional capacity of elderly residents of communities with a rapid population aging rate. Revista Brasileira de Geriatria e Gerontologia, 19(4), 643-652. https://doi.org/10.1590/1809-98232016019.150156

Borel, L. \& Alescio-Lautier, B. (2014). Posture and cognition in the elderly: Interaction and contribution to the rehabilitation strategies. Marseille, Neurophysiologie Clinique/Clinical Neurophysiology, 44(1), 95-107. https://doi.org/10.1016/j.neucli2013.10.129 
Cadore, E. L. et al. (2013). Effects of different exercise interventions on risk of falls, gait ability, and balance in physically frail older adults: a systematic review. Rejuvenation Research, 16(2), 105-114. https://doi.org/ 10.1089/rej.2012.1397

Camargos, F. F. et al. (2010). Adaptação transcultural e avaliação das propriedades psicométricas da Falls Efficacy Scale-International em idosos brasileiros (FES-I-BRAZIL). Revista Brasileira de Fisioterapia, 14(3), 237-243. https://doi.org/10.1590/S1413-35552010000300010

Cardoso, E., Dietrich, T. P \& Souza, A. P. (2021). Envelhecimento da população e desigualdade. Brazilian Journal of Political Economy, 41(1), 23-43. https://doi.org/10.1590/0101-31572021-3068

Castagno, L. A. (1994). Distúrbio do Equilíbrio: Um Protocolo de Investigação Racional - parte 2. Brazilian Journal of Otorhinolaryngology, 60 (2), $124-141$.

Cruz, D. E., Duque, R. O. \& Leite, I. C. G. (2017). Prevalence of fear of falling, in a sample of elderly adults in the community. Revista Brasileira de Geriatria e Gerontologia, 20 (3), 309-318. https://doi.org/10.1590/1981-22562017020.160176

Delbaere, K. et al. (2010). The Falls Efficacy Scale International (FES-I). A comprehensive longitudinal validation study. Age and Ageing, 39(2), 210-216. https://doi.org/10.1093/ageing/afp225

Fiorio, G. O., \& Meneghini, G. O. (2018). Functionality and risk of falls of elderly participants of a companionship group of Flores da Cunha, RS. PAJAR Pan American Journal of Aging Research, 6(2), 50-57. https://doi.org/10.15448/2357-9641.2018.2.31375

Galisteu, K. J. et al. (2006). Qualidade de Vida de idosos de um grupo de convivência com a mensuração da escala de Flanagan. Arquivos Ciências da Saúde, 13(4), 209-214

Gauchard, G. C. et al. (2003). Physical activity improves gaze and posture control in the elderly. Neuroscience Research, 45(4), 409-417. https://doi.org/10.1016/s0168-0102(03)00008-7.

Gillespie, L. D., et al. (2012). Interventions for preventing falls in older people living in the community. Cochrane Database Systematic Review,12(9), CD007146, sep./2012. https://doi.org/ 10.1002/14651858.CD007146.pub3.

Guimarães, L. H. C. T. et al. (2004). Comparação da propensão de quedas entre idosos que praticam atividade física e idosos sedentários. Revista Neurociências, 12(2), 68-72.

Hauser, E. et al._(2015). Fear of falling and physical performance in elderly practitioners of physical activity. Revista da Educação Física/UEM, 26(4), 593600. https://doi.org/10.4025/reveducfis.v26i4.28442

Hauser, E., Cardoso, L. F. \& Mazo, G. Z. Reference values for balance in physically active eldely women. Revista Brasileira de Medicina do Esporte, 26(4), 328-331. https://doi.org/10.1590/1517-869220202604193086

Horniak, V. et al. (2013). What is the relation between fear of falling and physical activity in older adults? Archives of Physical Medicine and Rehabilitation, 94(12), 2529-2534. https://doi.org/10.1016/j.apmr.2013.06.013

Hueb, M. M. \& Feliciano, C. P. (2012). Avaliação diagnóstica das síndromes vertiginosas. Revista Hospital Universitário Pedro Ernesto, 11(3), 23-27.

IBGE:Instituto Brasileiro de Geografica e Estatística. Síntese de Indicadores Sociais 2015 (2015). <https://biblioteca.ibge.gov.br/visualiza cao/livros/liv95011.pdf>

Jin, K. et al. (2015). The critical need to promote research of aging and aging-related diseases to improve health and longevity of the elderly population. Aging and Disease, 6(1), 1-5. https://doi.org/10.14336/AD.2014.1210

Kirk-Sanchez, N. J., \& McGough, E. L. (2014). Physical exercise and cognitive performance in the elderly: current perspectives. Clinical interventions in Aging, 9, 51-62. https://doi.org/10.2147/CIA.S39506

Lee, H. C. et al. (2013). Effects of a multifactorial fall prevention program on fall incidence and physical function in community-dwelling older adults with risk of falls. Archives of Physical Medicine and Rehabilitation, 94(4), 606-615. https://doi.org/10.1016/j.apmr.2012.11.037

Lopes, K. T, Costa, D. F, Santos, L. F., Castro, D. P. \& Bastone, A. C. (2009). Prevalência do medo de cair em uma população de idosos da comunidade e sua correlação com mobilidade, equilíbrio dinâmico, risco e histórico de quedas. Revista Brasileria de Fisioterapia, 13(3), 223-229.

Müjdeci, B., Aksoy, S. \& Atas, A. (2012). Evaluation of balance in fallers and non-fallers elderly. Brazilian Journal of Otorhinolaryngology, 78(5), 104-109. https://doi.org/10.5935/1808-8694.20120016

Myers, A. H., Young, Y. \& Langlois, J. A. (1996). Prevention of falls in the elderly. Bone, 18(1), S87-S101. https://doi.org/10.1016/8756-3282(95)00384-3

Pedrinelli, A, Garcez-Leme, L. E. \& Nobre, R. S. A. (2009). O efeito da atividade física no aparelho locomotor do idoso. Revista Brasileira de Ortopedia, 44(2), 96-101. https://doi.org/10.1590/S0102-36162009000200002

Peterka, R. J. (2002). Sensorimotor integration in human postural control. Journal of Neurophysiology, 88(3), 1097-1118. https://doi.org/ 10.1152/jn.2002.88.3.1097

PROCEMPA. População idosa de Porto Alegre $\quad-\quad$ informação demográfica e socioeconômica. 2015. 〈http://lproweb.procempa.com.br/pmpa/prefpoa/observatorio/usu_doc/informacao_demografica_e_socioeconomica-populacao_idosa02.pdf〉.

Rubin, A. S. B. (2002) Estudo do equilíbrio corporal de pacientes com catarata senil antes e após o tratamento cirúrgico. Dissertação (Mestrado em Distúrbios da Comunicaçao Humana) - Universidade Federal de Santa Maria, Santa Maria.

Ruschel, N. L. et.al. (2019). Posturografia foam-laser em idosos praticantes de exercícios físicos: comparação com dados normativos para adultos. Estudos Intesdisciplinares sobre o Envelhecimento, 24, 137-148. 
Research, Society and Development, v. 10, n. 7, e49110716794, 2021

(CC BY 4.0) | ISSN 2525-3409 | DOI: http://dx.doi.org/10.33448/rsd-v10i7.16794

Ruwer, S. L. (2002). Estudo da Posturografia Dinâmica Foam Laser em indivíduos normais com idades entre 14 e 60 anos. 2006. $87 f$. Dissertação (Mestrado em Distúrbios da Comunicação Humana) - Universidade Federal de Santa Maria, Santa Maria.

Sandoval, R. A et al. (2013). Ocorrência de quedas em idosos não institucionalizados. Revista Brasileira de Geriatria e Gerontologia, 16(4), 855-863. https://doi.org/10.1590/S1809-98232013000400019

Scianni, A. A. et al. (2019). Efeitos do exercício físico no sistema nervoso do indivíduo idoso e suas consequências funcionais. Revista Brasileira de Ciências do Esporte, 41(1), 81-95. https://doi.org/10.1016/j.rbce.2018.03.026

Siqueira, F. V. et al. (2011). Prevalence of falls in elderly in Brazil: a countrywide analysis. Cadernos de Saúde Pública, 27(9), 1819-1826. https://doi.org/10.1590/S0102-311X2011000900015

Soldera, C.L.C. (2013). Participação dos sistemas de manutenção do equilíbrio corporal, do risco de quedas e do medo de cair em idosos e longevos. 2013. 152f. Tese (Doutorado em Gerontologia Biomédica) - Pontifícia Universidade Católica do Rio Grande do Sul, Porto Alegre.

Teixeira, A. R., Cardoso, M. de M. \& Olchik, M. R. (2021). Idade subjetiva em idosos ativos: estudo comparativo com idade cronológica, aspectos sociodemográficos e autoavaliação de saúde. PAJAR - Pan American Journal of Aging Research,9(1), e39344. https://doi.org/10.15448/23579641.2020 .1 .39344

Teixeira, C. L. (2013). Equilbrio e controle postural. Brazilian Journal of Biomechanics, 11(20), 30-40.

Teixeira, C. S. et al. (2015). Equilíbrio postural: investigação com crianças, adultos e idosos. Revista Brasileira de Ciências do Envelhecimento Humano, 12(2), 134-146. https://doi.org/10.5335/rbceh.v12i2.5158

Teixeira-Leite, H. \& Manhães, A. C. (2012). Association between functional alterations of senescence and senility and disorders of gait and balance. Clinics, 67(7), 719-729. https://10.6061/clinics/2012(07)04

Tinetti, M.E., Richman, D. \& Powell, L. (1990). Falls efficacy as a measure of fear of falling. Journal of Gerontology, 45(6), $239-243$. https://doi.org/10.1093/geronj/45.6.p239.

United Nations. (2019). World Population Ageing 2019: highlights.

Vieira L.S. et al. (2018). Falls among older adults in the South of the Brazil: prevalence and determinants. Revista de Saúde Pública, 52, e-22. https://doi.org/10.11606/S1518-8787.2018052000103

Yardley L. et al. (2005). Development and initial validation of the falls efficacy scale-international (FES-I). Age and Ageing, 34(6), 614-619. https://doi.org/10.1093/ageing/afi196. 